\title{
i-ZEN an Intelligent Zero ENergy Flow Meter
}

\author{
Anastasios Fanariotis, Theofanis \\ Orphanoudakis \\ Hellenic Open University \\ Tsamadou 13-15, GR-26222, Patras, GREECE \\ \{a.fanariotis, fanis\}@eap.gr
}

\author{
Efthimios Pariotis \\ Hellenic Naval Academy \\ Hadjikyriakou Av. Piraeus, Athens, Greece \\ pariotis@snd.edu.gr
}

\begin{abstract}
In this paper we present a fully energy autonomous water flow meter exploiting a low power controller and communications module in order to integrate the resulting device into an intelligent zero (externally provided) energy (i-ZEN) flow meter for the Internet of Things. We describe the optimized for power consumption hardware design, the application to exploit the collected data and we present measurements from the evaluation of the prototype in operation that show that the device can operate practically for unlimited time.
\end{abstract}

Keywords-energy harvesting, low energy, flow meter, Bluetooth, IoT

\section{INTRODUCTION}

Accurate near real time water flow measurement is vital both for reasons of water preservation as well as reduction of water charges. State of the art Internet of Things (IoT) technologies present an attractive solution since they deliver platforms that can serve both as sensors, actuators and communication devices. IoT devices can thus become enablers for remote water flow monitoring and control over the Internet provided that energy sources are available to provide the necessary power for the device microcontroller and active electronic components. Since water meter installations generally bring up sever difficulties for connecting to electricity distribution networks the energy autonomous operation of next generation networked smart meters is a hard requirement.

To address this requirement, devices need to operate either powered by batteries or by means of appropriate renewable sources that can be easily installed in the vicinity of the water flow meter. Then the water flow meter reading must be performed by the active device, which in turn must process the received data, encapsulate them implementing appropriate network protocol stacks and transmit them to a remote endpoint so that they can be easily managed by users that have authorized access to them.

In this paper we present a solution based on an energy harvesting power generator to supply the necessary power for autonomous operation of a measurement-transmission unit integrated to a water flow meter exploiting the widely adopted Bluetooth 4.2 (also known as Bluetooth Low Energy, BLE) wireless communication standard. In the following sections we first present the design considerations and practical limitations in relation to prior works in this field, then we describe the implementation of a hardware and software prototype based on commercially available components that can meet the design specifications and finally we present evaluation results focusing on power consumption that demonstrate that the device can operate practically for unlimited time.

\section{PROBLEM STATEMENT AND PRIOR WORK}

Following the previous introduction, we can summarize the basic requirements of a smart energy autonomous water flow meter stating that it should:

- operate sufficiently based solely on its own power supply or power generation unit

- perform accurate near real time water flow measurement

- provide an interoperable wireless interface for remote connection and reading

- exploit state-of-the-art IoT technologies and infrastructures

- $\quad$ operate unattended as long as possible

While battery operated devices could be considered as an option to satisfy autonomous operation current battery capacities cannot guarantee adequate lifetime taking into account the power consumption requirements of the device. Thus, rechargeable batteries exploiting energy harvesting methods [1], [2] have been recently proposed. The authors in [1] exploit a turbine generator with moving blades rotated by the water flow itself, which is the same principle of operation used for water flow measurement by means of a magnetic Hall-effect sensor measuring the frequency of blade revolutions. The device presented in [1] shares some similar principles with i-ZEN but communication is based on the Wireless M-Bus standard, which limits interoperability with IoT, requiring direct communication with compatible only wireless devices and trades off measurement accuracy for power consumption. While the principle of power generation is presented in [1] no results are presented with respect to power consumption and device autonomy under different conditions. The same wireless M-Bus interface is used in [3], while a mobile communications GSM module is used in the open source project described in [4]. The device described in [3] operates only on batteries with no power generation unit, limiting its power budget and functionality, and employs a piezoelectric flow sensor,

ISBN 978-80-261-0722-4, (C) University of West Bohemia, 2018

This research was partially privately funded by Deon Energy ltd, 35 Grafton Way, London, WIT 5 DB, Office, 573 Basingstoke Reading, Rg20sj. 
without requiring any rotating parts. The device described in [4] additionally features an electrically operated water valve that combined to the GSM module greatly increase its power consumption requirements, while the actuation control ins based on AI/ML methods through Big Data analysis. This adds an extra layer of complexity during initial setup and the need for additional user-correction actions during the period where not enough captured data is available.

\section{SYSTEM DESCRIPTION}

The system described in this paper is designed as an autonomous/unattended water flow/consumption measurement unit with cloud storage capabilities and data visualization in order to be user-friendly. Since a lot of work has been done in the area of water flow energy harvesting this paper is steered towards the minimization of hardware needed and thus the cost of implementation and the maximization of power efficiency and service intervals. It is based on a heterogeneous network consisting of two types of communications stacks (BLE \& TCP/IP) and it is designed to be power efficient and service-free limited only by the service life of the battery contained.

\section{A. System Architecture}

The system consists of two main subsystems, the measurement-transmission unit and the concentratorgateway system. The first is based on a low power System-on-Chip (SoC), a power generator with energy storage capabilities and a flow meter. The power needed from both the $\mathrm{SoC}$ and the flow meter is extracted from the water flow and stored to an onboard LiPo battery to the power generator.

The selected SoC is an ARM based microcontroller module with RF capabilities and the flow sensor is based on a hall-effect sensor element that creates a square wave signal with $50 \%$ duty cycle and frequency proportional to the water flow. The SoC module calculates the instant flow and total volume from this signal and merges them with secondary captured data (battery voltage level and estimated power consumption) into data packets that are transmitted in preselected time intervals by means of BLE protocol and usage of the notify function on a vendor specific (custom) channel/GATT. The device is set-up as a BLE peripheral and thus is capable to accept connections from both the gateway and various devices with BLE capabilities, like smart phones.

The gateway unit runs on linux, it is powered from the mains electrical system as an in-premises device and utilizes both the BLE stack and $\mathrm{WiFi} / \mathrm{LAN}$ connections towards the internet. The in-house software developed runs on a Raspberry Pi board with pre-installed BLE communications software and exports any captured data to a local TCP socket implementing in such way interprocess communication towards the application that translates the captured data to a format acceptable by the selected cloud service. As such service thingspeak (https://thingspeak.com/) was selected in order to verify the functionality and accuracy of the system. This type of architecture (Figure 1) was selected in

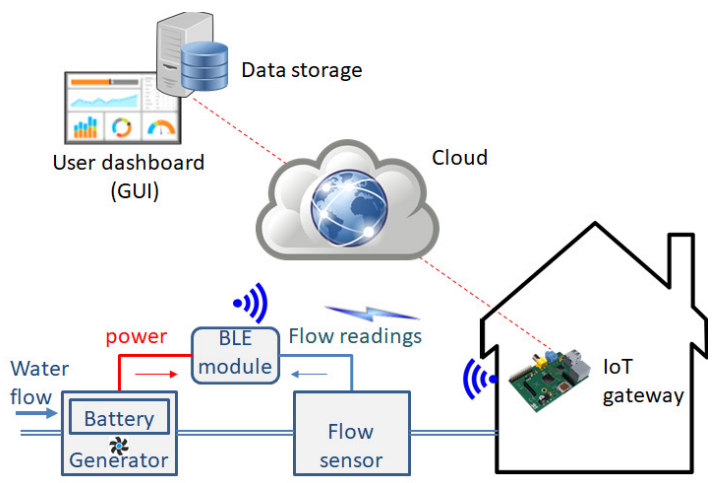

Figure 1. System block diagram

order to alleviate any heterogeneity problems and keep the scalability of the system good as a distributed system.

\section{B. Software Environment}

The software developed for the system is totally transparent to the user, follows the design rules of distributed systems and is composed by three software packages.

The firmware, was developed in $\mathrm{C}$ language, it runs on the BLE/MCU module and is responsible for the power management, the measurements and the transmission of data towards the gateway. It is completely transparent to the user and can be adjusted during the initial set-up with respect to the functionality of BLE addressing, data transmission intervals and flow sensor calibration.

The middleware runs on an in-premises Raspberry Pi and it was developed on Java as a multi-platform software package. It is totally transparent to the user as well and no other user-actions are needed other than the initiation/run of the software.

The last software package consists of a third-party cloud service that offers an intuitive GUI and stores the captured data for later usage or statistical processing if needed. The GUIs main function is to display the water flow and the total volume used per date/time accompanied by the device state data of current Battery voltage level and estimated instant Power Consumption. An instance of the GUI is shown below (Fig. 2), captured when the system was under development at 20 Feb 2018 and powered by a bench power supply at $3.3 \mathrm{~V}$. All the measurements and tests made towards the GUI are publicly available at https://thingspeak.com/channels/396539/.

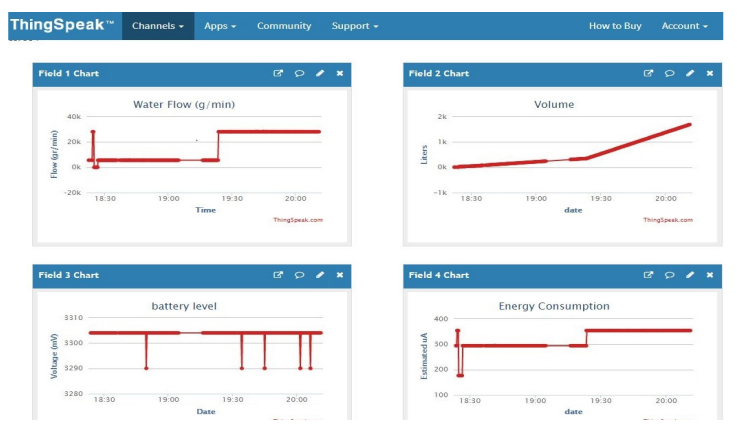

Figure 2. Thingspeak GUI snapshot 


\section{EXPERIMENTAL SETUP}

For experimental purposes, a set of commercial available devices was selected (Fig. 3). First as a SoC module the nordic nRF51822 was selected that it is based on an ARM Cortex M0 and provides an SDK with support for BLE stack (S130) with low power capabilities. As a power generator and energy storage unit the DB-368 ZhongJiang Micro-Hydro generator was selected that it is capable of $125 \mathrm{~mW}$ power production and has an integrated $800 \mathrm{mAh} \mathrm{LiPo}$ battery. This unit offered a stable voltage output of 3.6 $\mathrm{V}$ and an onboard recharge circuit. Lastly, a flow meter was selected (YF-S201) from the same company that was based on a hall-sensor element capable of functioning correctly in the voltage range of $3.6 \mathrm{~V}$ (WSH130:2.4-26V). However, the original hallsensing element did not provide low power operation ( $2 \mathrm{~mA}$ current draw according to the datasheet) so it had to be substituted with an ultra low power element from Texas Instruments (DRV5012) with current draw of $155 \mu \mathrm{A}$ at high speed setting in order to reach our power budget objectives. In order to detect the battery voltage level a high resistance voltage divider accompanied by a charge transfer capacitor for lowfrequency sampling by the SoCs ADC was used. Finally, for measurement validation reasons, a benchtype calibrated hydraulic system was developed capable of creating accurate water flow. The experimental setup was then connected to this system in order to verify that the flow measurement results were within the real flow values produced by the hydraulic system.

\section{PERFORMANCE EVAluation RESUlts}

All the performance evaluation measurements were made towards the functionality and autonomy of the system. The main target was to prove the capability of the system to function under long periods of time without any power generation while keeping the energy consumption lower than the energy generation when there was sufficient water flow. The measurements taken are described in this chapter. For the current draw measurements of the BLE module a shunt resistor of $10 \Omega$ was used since measurements were within the range of $20 \mu \mathrm{A}$ to instances of $17 \mathrm{~mA}$. A measurement instance of the BLE unit transmitting as captured by the oscilloscope measuring voltage drop vs. time at the BLE's power input is shown in Fig. 4.

\section{A. Flow and Energy Production Measurements}

According to its datasheet, the power generator is capable of producing $125 \mathrm{~mW}$ of power output with a minimum flow of 4 liters per minute (lpm)

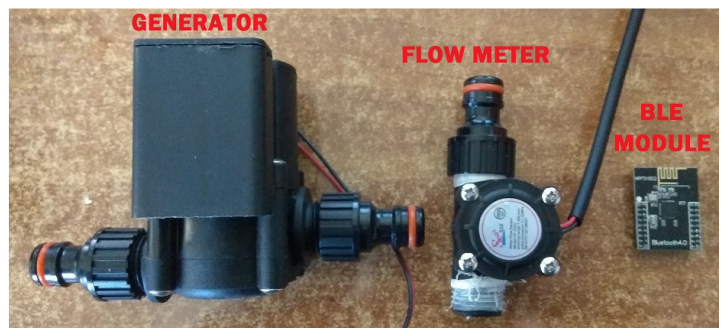

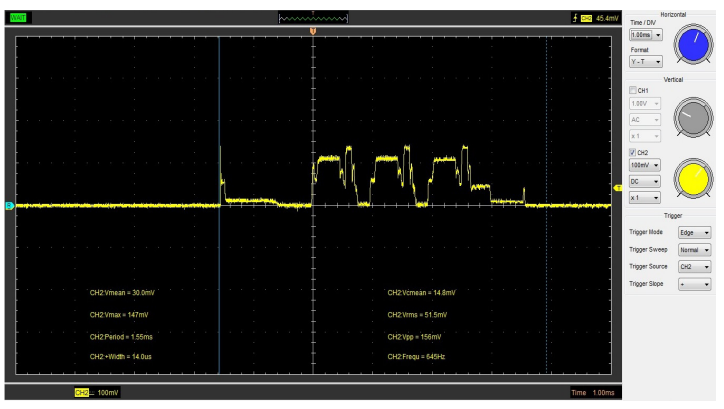

Figure 3. BLE Instance of transmission

consistently over the entire range of its flow capability. After analyzing the circuit contained in the device this is found to be correct since a zener-type voltage regulation unit is used dissipating any extra generated energy to the environment. While this is an energy inefficient design, it deemed to be suitable for the system power requirements since it can provide a continuous current of at least $34.72 \mathrm{~mA}$ with a suitable load connected. According to the device's datasheet the power production per liter of water is indicated in Figure 4.

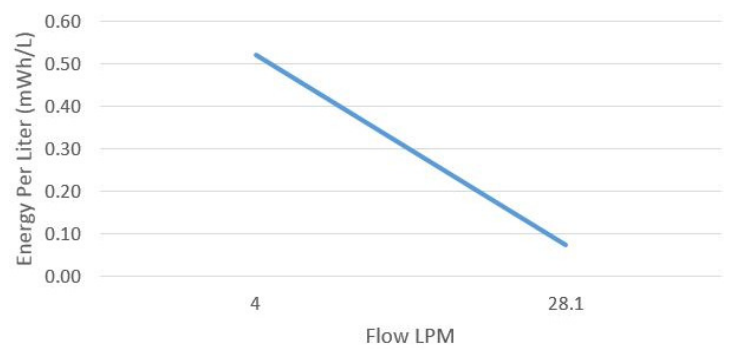

Figure 4 . Energy per liter of water

\section{B. Power consumption vs. battery lifetime}

The system's power consumption was evaluated by measuring separately the consumption of three sub-systems: the voltage divider, the flow sensor and the SoCs consumption under various states. The voltage divider with a total resistance of $15 \mathrm{M} \Omega$ had a $0.24 \mu \mathrm{A}$ current draw at $3.6 \mathrm{~V}$, the modified flow sensor given its push-pull type output and in accordance with the datasheet was contributing to the total current draw an additional $155 \mu \mathrm{A}$ under all conditions and finally the $\mathrm{SoC}$ was measured under different states since the power consumption was affected by the sensor interrupt rate and the connection state of the BLE (Table 1).

The total system autonomy was estimated taking into account the sum of the average current draw from all the subsystems and a usable battery capacity of $800 \mathrm{mAh}$ (Table 2). For comparison reasons, the calculated autonomy of SoC without the flow meter connected is also given in the same table. As indicated in Table 2 the amount of power generated is more than the total system power consumption; thus, system autonomy approaches infinity (indicated as "Inf" in Table 2) as long as enough water flow is present. 
TABLE 1. SYSTEM CURRENT DRAW

\begin{tabular}{|l|c|c|c|c|c|}
\hline \multirow{2}{*}{$\begin{array}{c}\text { Flow } \\
\text { lpm }\end{array}$} & \multicolumn{5}{|c|}{ Average current draw $(\boldsymbol{\mu A})$} \\
\cline { 2 - 6 } & $\begin{array}{c}\text { Advertising } \\
\text { (1.2 sec } \\
\text { interval) }\end{array}$ & $\begin{array}{c}\text { Connected } \\
\text { (1 sec data } \\
\text { send interval) }\end{array}$ & Vd & $\begin{array}{c}\text { Hall } \\
\text { sens }\end{array}$ & $\begin{array}{c}\text { Total } \\
\text { (connected } \\
\text { state })\end{array}$ \\
\hline 0 & 25.16 & 21.06 & 0.24 & 155 & 176.3 \\
\hline 5.5 & 139 & 141 & 0.24 & 155 & 296.2 \\
\hline 28.1 & 203 & 200 & 0.24 & 155 & 355.2 \\
\hline
\end{tabular}

TABLE 2. SYSTEM AUTONOMY

\begin{tabular}{|l|c|c|c|}
\hline \multirow{2}{*}{ System } & \multicolumn{3}{|c|}{ Autonomy (Days) } \\
\cline { 2 - 4 } & 0 flow & 4 lpm flow & 28.1 lpm flow \\
\hline No hall sensor & 1528.78 & Inf & Inf \\
\hline Full device & 189.07 & Inf & Inf \\
\hline
\end{tabular}

In order to achieve a better grasp of the power generation and requirements of the system in real world values, the results were reduced to autonomy time vs. volume of water and time required for recharge as shown in Table 3 . In this scenario, the device was connected to the BLE gateway, transmitting for $24 \mathrm{~h}$ without any power generation/harvesting from water flow.

TABLE 3. 24H ENERGY BALANCE

\begin{tabular}{|l|l|}
\hline \multicolumn{1}{|c|}{ Calculation } & Value \\
\hline Energy Consumed (mWh) & 15.23 \\
\hline Minimum Volume Needed for Recharge (1) & 29.5 \\
\hline Maximum Volume Needed for Recharge (1) & 207.6 \\
\hline Time Needed (minutes with Flow $>4$ lpm) & 7.375 \\
\hline
\end{tabular}

\section{CONCLUSIONS \& FUTURE WORK}

In this paper, an autonomous energy-efficient, cloud-enabled water flow meter was described. Through the measurements the autonomy and functionality was proven to be excellent. Although it was not the aim of this paper to analyze the efficiency of the generator itself, the energy generated per liter of water (Fig. 5) raised some questions about the design of the voltage regulation circuit inside the generator. After close examination this was found to be designed around a single zener-type voltage regulator circuit that created two problems, firstly very low-efficiency of power harvesting/generation, especially for very small loads and high flow of water and secondly inability of utilization of the full battery capacity because of the charging voltage level being lower than optimal. In this area a multistage voltage regulator design with multiple low-quiescent current switching regulators or high efficiency LDO regulators design may improve energy harvesting, storage and system autonomy dramatically by reducing the needed battery charge time when flow of water is high enough and utilizing the full battery capacity.

As it is described in this paper it was decided that the replacement of the hall-sensor element in the flow meter unit was beneficial, but, even with this improvement, the current draw of $155 \mu \mathrm{A}$ can be considered as excessive for low power applications. The usage of a mechanical (reed) sensor was out of the question because of the high failure rate so in order to improve power efficiency more research is needed in order to use the DRV5012 hall-sensor element in low speed mode (current draw $1.6 \mu \mathrm{A}$ ) during no-flow conditions and then switch it to high-speed mode when flow is detected. This however presents the problem of the initial signal sampling aliasing and there may be occasions where no signal is detected by the flow meter. In order to implement such a method a mechanical match of the turbine wheel characteristics (acceleration, rate of acceleration etc.) versus the flow of water and generated signal frequency must be researched in order to minimize or alleviate any signal aliasing. In the same area the flow sensor unit may be completely eliminated by placing the hall sensor element inside the generator, however this may present the problem of flow measuring errors during the generator turbine speed slowing down under high electrical loads. In order to implement such a method a full mapping of generator speed vs electrical load and water flow is needed.

Lastly, in the presented application a BLE module based on Bluetooth 4.2 was used. While its capabilities were deemed and proved to be enough, newer modules with better hardware that support Bluetooth 5 with newer abilities and characteristics such as lower power consumption and long range of signal transmission may add value to this application, making it more useful and even more efficient.

\section{REFERENCES}

[1] P. Becker et al., "Energy Autonomous Wireless Water Meter with Integrated Turbine Driven Energy Harvester", 2013 J. Phys.: Conf. Ser. 476012046

[2] S.-H. Kwon, "An effective energy harvesting method from a natural water motion active transducer", Energy Environ. Sci., 2014,7, 3279-3283

[3] Kamstrup A/S, flowIQ ${ }^{\circledR} 3100$ Water Meter, Technical Description, 5512-1242_B1_GB 05.2015

[4] E. Kestler, Congnitive GSM Autonomous Water Meter, https://www.hackster.io/flatbox/congnitive-gsm-autonomouswater-meter-71762f 